\title{
Multimodel biometrics Fusion based on FAR and FRR using Triangular Norm
}

\author{
Di Wu ${ }^{* 1,2}$ \\ ${ }^{1}$ college of Electrical and Information, Hunan Institute of Engineering, Xiangtan, 411004, China \\ ${ }^{2}$ College of Computer and Communication, Lanzhou University of Technology, Lanzhou, 730050, China \\ Jie Cao \\ College of Computer and Communication, Lanzhou University of Technology,Lanzhou, 730050, China \\ E-mail: caoj@lut.cn
}

Received 13 October 2014

Accepted 16 May 2015

\begin{abstract}
Multibiomitric systems are expected to be more accurate due to the presence of multiple evidences, score level fusion is the most commonly used approach in multibiometrics. In this paper, A novel approach is proposed for the fusion at score level fusion based on False Reject Rate(FRR) and False Accept Rate(FRR) using triangular norms(t-norms). This study aims at tapping the potential of t-norms for information fusion at first, at the second, it transfers scores into Transfer function based on corresponding FRRs and FARs, thus avoiding calculating posterior probability of a certain score. Experiment result shown that the proposed method renders very good performance as it is quite computationally and outperforms the traditional score level fusion schemes, the experimental result also confirms the effectiveness of the proposed method to improve the performance of multibiometric system.
\end{abstract}

Keywords: MultiBiometrics, Score Level Fusion, False Reject Rate(FRR), False Accept Rate(FRR), triangular norms, Transfer function

\section{Introduction}

The problem of automatic recognition of individuals using biometrics has received a great deal of interest in the last few decades[1]. This is mainly due to the obvious advantages that biometrics has to offer over conventional means of identity verification. However, whilst

\footnotetext{
${ }^{1}$ Corresponding Author: wudi6152007@163.com

This work was supported by in part the National Science-technology Support Plan Project of China under contract 1214ZGA008, in part by the Nature Science Foundation of China under contract 61263031, in part by the Science Foundation of Gansu Province of China under contract 1010RJZA046.
}

considerable advance have been achieved in this area, unimodal biometric systems are plagued by several drawbacks, such as susceptibility of the result to the quality of the sample, its orientation/rotation and distortion, noise, intra-class variability, risk of spoofing and others[2]. Confronted by the limitation of unimodal systems, we are gravitated toward multibiometric sources which utilize the evidences presented by multiple biometric sources in order to determine or confirm the identity of an individual.

According to the level of information fused, we can subdivide them in five categories [3]: sensor level fusion, feature level fusion, matching level fusion, rank level 
fusion and decision level fusion. Among them, score level fusion is the most frequently utilized because of easy availability of the scores and contains ample information to discriminate between genuine and impostor scores. Already existing score level fusion approaches can be categorized into three classes: transformation-based, density-based and classifier-based.

The first score level fusion method is known as transformation-based[4], in practice, owing to the limited availability of training data, accurate estimation of the joint conditional densities for all classes is not always possible. In such situations, a more appropriate fusion method is to directly combine the scores provided by different matches without converting them into a posterior probabilities. Score normalization is needed to transform the scores obtained from different classifier into a common domain, consequently, in the transformed domain, the sum, max and min combination rules can be directly applied. In the density-based fusion method[5] , the matching scores are transformed into posterior probability at first, then the Bayes rules are used to the final decision. The density functions of the match score are usually not known and have to be estimated from a set of training scores of the classes. Density estimation can be accomplished either by the parametric or non-parametric methods. In the classifier-based score level fusion[6], it takes $\mathrm{N}$ matching scores as an $\mathrm{N}$-dimensional feature vector that is used to categorize into one of two possible classes: genuine and impostor. Based on the training data, the classifier learns a decision boundary between the classes.

In conclusion, the transformation-based fusion approach, with no training process and few consideration of distribution of matching scores and it is easy to implement[7]. On the contrary, density-based fusion method, which requires accurate estimation of density and huge number of training samples, is hard to carry out for the following reasons: firstly, positive samples, namely genuine matching scores are limited in today's multi-biometric systems, secondly, it is difficult to estimate the density of matching scores in that they may not obey a certain distribution model. Classifier-based fusion has its advantage of freeing from the restriction of different distribution of matching scores, but it needs sufficient training samples and new training for a different system.

Based on the above analysis of the advantages and disadvantages of existing methods, we propose a new approach to describe the distribution of matching scores by FRR and FAR, where they are two important parameters of illustrating performance of a recognition system. In order to enhancing the performance of score level fusion, we investigate t-norm using for the score level fusion, these norms deal with the real challenge of uncertainty and imperfection pervading the different sources of knowledge.

In this paper, a new score level fusion method is propose which based on FRR and FAR using t-norm. It firstly calculates FRR and FAR based on training samples, and obtained the Transfer function based on FAR and FRR. Then computes Transfer function of each matching scores through interpolation based on testing samples. At the last, the final decision are made by t-norms. Compared to the existing methods, the fusion algorithm proposed in this paper can greatly improve the recognition performance of multibiometric system.

The remainder of this paper is organized as follows: The t-norms is discussed in Section 2. The scheme which transform the score to Transfer function based on FRR and FAR is described in Section 3. In Section 4, the new fusion method named transfer function triangular fusion(TFTF) is briefly discussed. Hence, the experiments result is presented in Section5. Section 6 conclude our work and outlines our future work to generalize the method to multibiometric recognition system.

\section{Preliminaries of t-norms}

Triangular norms(t-norms) and t-conorms are the most general families of binary functions that satisfy the requirements of the conjunction and disjunction operators respectively[8]. These t-norms $T\left(S_{1}, S_{2}\right)$ and t-conorms $S\left(S_{1}, S_{2}\right)$ are two place functions that map the unit square into the unit interval, i.e., $T\left(S_{1}, S_{2}\right):[0,1] \times[0,1] \rightarrow[0,1] \quad$ and $S\left(S_{1}, S_{2}\right):[0,1] \times[0,1] \rightarrow[0,1]$.

A t-norms is a function $\mathrm{T}:[0,1] \times[0,1] \rightarrow[0,1]$ which satisfies the following properties[9]:

(1) Commutativity: $T\left(S_{1}, S_{2}\right)=T\left(S_{2}, S_{1}\right)$

(2) Monotonicity: $\quad T\left(S_{1}, S_{2}\right) \leq T\left(S_{3}, S_{4}\right)$ if 
(3) Associativity: $T\left(S_{1}, T\left(S_{2}, S_{3}\right)=T\left(\left(S_{1}, S_{2}\right), S_{3}\right)\right.$

(4) $T\left(S_{1}, 1\right)=S_{1}$

Specifically, t-norms do not require the assumption of evidential independence of the modalities to be fused. As t-norms are associative, fusion of three or more modalities can be done irrespective of the order. Their corresponding boundary condition, the evaluation of the t-norms at the extremes of the [0,1] interval, satisfy the truth tables of the logical AND and OR operators. T-norms are a generalization of the usual two-valued logical conjunction. The monotonicity property of the t-norms ensure that the degree of truth of conjunction does not decrease if the truth values of conjunct increase. Continuity, which is often required from the fuzzy conjunction point of view requires that very small changes in the truth values of conjunct should not drastically affect the truth value of their conjunction.

The most used t-norms are taken as follows[10]:

$$
\begin{aligned}
& \text { Einstein product: } \frac{S_{1} S_{2}}{2-\left(S_{1}+S_{2}-S_{1} S_{2}\right)} \\
& \text { Hamacher: } \frac{S_{1} S_{2}}{S_{1}+S_{2}-S_{1} S_{2}} \\
& \text { Yager: } \max \left(1-\left(\left(1-S_{1}\right)^{p}+\left(1-S_{2}\right)^{p}\right)^{1 / p}, 0\right)
\end{aligned}
$$

\section{Compute transfer function based on FRR and FAR}

Matching scores coming different recognition systems are not comparable directly, at the same time, FRR and FAR can be obtained by setting threshold as each score. Therefore, we firstly calculate FRR and FAR of each matching score based on training samples, then obtained Transfer function based on FRR and FAR which coming from training samples, at last, we compute Transfer function of each matching score through interpolation based on testing samples.

\subsection{FRR and FAR computation based on training samples}

Suppose an two fusion system has $\mathrm{N}$ training samples. For the ith training samples, the template feature is $U=\left[u_{i}^{1}, u_{i}^{2}\right]$, input feature is $V=\left[v_{i}^{1}, v_{i}^{2}\right]$, the matching score is $S=\left[s_{i}^{1}, s_{i}^{2}\right]$. In addition, we use $z_{i}$ to note the ith training samples whether genuine or impostor. When the training samples is belong to genuine, then $z_{i}$ is 1 ; otherwise the training samples is belong to impostor, then $z_{i}$ is 0 . So the FRR and FAR of the matcher based on training samples are respectively calculated as follows[11-12]:

$$
\begin{gathered}
F A R(t)=P(s \geq t \mid \operatorname{Im} \text { postor })=\frac{\left|\left\{i \mid s_{i} \geq t, z_{i}=0\right\}\right|}{\sum_{i=1}^{N}\left(1-z_{i}\right)}(4) \\
F R R(t)=P(s<t \mid \text { Genuine })=\frac{\left|\left\{i \mid s_{i}<t, z_{i}=1\right\}\right|}{\sum_{i=1}^{N} z_{i}}(5)
\end{gathered}
$$

\subsection{Transfer function calculation based on FRR and FAR belong to training samples}

It is show that the FRR and FAR are the variables which calculate the a range of error rate and correct rate and accumulate the error rate of each matching point. It is reflect the distribution of the matching scores in the training set, avoiding compute the error rate or correct rate to a specific matching score. Form the viewpoint discussed above, we know it is difficult to compute the ideal transfer function. So in this paper, we construct a new transfer function based on FRR and FAR using confidence function.

The first step is compute confidence function $B_{k}(t)$ using equation (6):

$$
\begin{gathered}
B_{k}(t)= \begin{cases}\frac{F R R_{0}}{F A R_{k}(t)} & F R_{k}(t) \\
\frac{F A R_{0}}{F R R_{k}(t)} & F A R_{k}(t)=0 \\
\frac{F A R_{k}(t)}{F A R_{k}(t)} & \text { else }\end{cases} \\
\sum_{i=1}^{N}\left(1-z_{i}\right)
\end{gathered}
$$

$$
F R_{k}(t)=\frac{\left|\left\{i \mid s_{i}^{k}<t, z_{i}=1\right\}\right|}{\sum_{i=1}^{N} z_{i}}
$$


In order to ensure the distinguish ability of the confidence function $B_{k}(t)$ When $F R R_{k}(t)$ or $F A R_{k}(t)$ are equal to zero, we define $F R R_{0}$ or $F A R_{0}$ in this paper and the calculation is taken as follows:

$$
\begin{aligned}
& F A R_{0}=\frac{1}{\sum_{i=1}^{N}\left(1-z_{i}\right)} \\
& F R R_{0}=\frac{1}{\sum_{i=1}^{N}\left(z_{i}\right)}
\end{aligned}
$$

For unimodal recognition system, the ordering of the confidence function is equal to the ordering of the probability of the matching score, that means the larger of the confidence degree, the larger of the probability of the matching result belong to the genuine is. From the equation (6), we can find that $B_{k}(t)$ is not within the range 0 and 1 ,Inspired by the idea of ideal transfer function $T_{\text {ideal }}$, we can obtain the transfer function $T_{k}(t)$ which based on confidence function $B_{k}(t)$ of the kth matcher[13]:

$$
T_{k}(t)=\frac{B_{k}(t)}{B_{k}(t)+1}
$$

From the equation (11), we know $T_{k}(x) \in[0,1]$ and we can further proof the following two equations:

$$
\begin{gathered}
x \leq y \Rightarrow B_{k}(x) \leq B_{k}(y) \\
T_{k}(x) \leq T_{k}(y) \Rightarrow B_{k}(x) \leq B_{k}(y)
\end{gathered}
$$

\subsection{Transfer function calculation based on FRR and FAR belong to tesing samples}

According to the above analysis, we known that the calculation of the transformation function based on FAR and FRR is difficult and fussy, it is needs all the matching scores which is impossible in a real recognition system, so we can only calculate it on the training set. Generally speaking, the training set and testing set have the same probability distribution, which means that if one score appears in the training samples, it will probably appear in the testing samples as well. So in this paper, we using interpolation method to obtain the transformation function value in the testing set.

Suppose in the training process, there $n(n<N)$ different matching scores are generated by $k t h$ matcher based on $N$ training samples and we ranking them in a ascending order as $\left\{t_{1}^{k}, t_{2}^{k}, \ldots, t_{n}^{k}\right\}$, then we can obtain $\left\{F A R_{k}\left(t_{i}^{k}\right) \mid 1 \leq i \leq n\right\}$ and $\left\{F R R_{k}\left(t_{i}^{k}\right) \mid 1 \leq i \leq n\right\}$ by equation( 7 ) and equation( 8 ). In the testing process, for the coming matching score $x$, the $F A R_{k}(x)$ and $F R R_{k}(x)$ are calculated using interpolation method in the four situations as follows:

(1) If $x<t_{1}^{k}$, that means all matching scores generated in the training process are larger than $x$, then we using $t_{1}^{k}$ and $t_{2}^{k}$ as control points, and calculate $T_{k}(x)$ as follows:

$$
T_{k}^{\prime}(x)=\frac{t_{2}^{k}-x}{t_{2}^{k}-t_{1}^{k}} \cdot T_{k}\left(t_{1}^{k}\right)+\frac{x-t_{1}^{k}}{t_{2}^{k}-t_{1}^{k}} \cdot T_{k}\left(t_{2}^{k}\right)
$$

(2) If $x>t_{n}^{k}$, that means all matching scores generated in the training process are samller than $x$, then we using $t_{n-1}^{k}$ and $t_{n}^{k}$ as control points, and calculate $T_{k}(x)$ as follows:

$$
T_{k}^{\prime}(x)=\frac{t_{n}^{k}-x}{t_{n}^{k}-t_{n-1}^{k}} \cdot T_{k}\left(t_{n-1}^{k}\right)+\frac{x-t_{n-1}^{k}}{t_{n}^{k}-t_{n-1}^{k}} \cdot T_{k}\left(t_{n}^{k}\right)
$$

(3) If there exist $x$ which equal to $t_{i}^{k}$, then

$$
T_{k}^{\prime}(x)=T_{k}\left(t_{i}^{k}\right)
$$

(4) If $t_{i}^{k}<x<t_{i+1}^{k}$, we can obtain $T_{k}(x)$ as follows:

$$
T_{k}^{\prime}(x)=\frac{t_{i+1}^{k}-x}{t_{i+1}^{k}-t_{i}^{k}} \cdot T_{k}\left(t_{i}^{k}\right)+\frac{x-t_{i}^{k}}{t_{i+1}^{k}-t_{i}^{k}} \cdot T_{k}\left(t_{i+1}^{k}\right)
$$

\section{The proposed new fusion method}

During score level fusion, score vector is combined to generate a single scalar score which is later used to make the final decision. Based on the Section 3, the matching score can be transformed to another score which is transfer function. All the training scores and testing scores can be transformed into their transfer function.

In this paper, for applying t-norms, the matching scores from all the modality are first converted to the common domain $[0,1]$ to guarantee a meaningful 
combination of the scores. The normalization criterion to do this is taken as[14]:

$$
x^{\prime}=\frac{x-\min (x)}{\max (x)-\min (x)}
$$

Where $x^{\prime}$ represents the normalized scores that are combined using the new method proposed in this paper. This kind of fusion is done in an associative manner,that means first combining the fusion output of the first two modalities with the third modality, and so on, until all the modalities are fused. If $T_{1}, T_{2}, T_{3}$ are the transfer functions of the three modalities, so the combined score $T$ is given by:

$$
\begin{gathered}
T=T\left(T_{3}, T\left(T_{1}, T_{2}\right)\right) \\
T(x, y)=\mathrm{t}-\operatorname{norm}(x, y)
\end{gathered}
$$

The steps of the training process is list as follows:

(1) Obtain the FAR and FRR in the training process of the single modality using equation (4) and (5);

(2) Calculate the confidence function $B_{k}(t)$ and transfer function $T_{k}(t)$ belong to corresponding FAR and FRR of the single modality the by equation (6) and (11).

The steps of the testing process is list as follows:

(1)Calculate the transfer function $T_{k}(t)$ in the testing process of the single modality using the testing matching score $x$ by equation (14) to (17);

(2) Fusion the $T_{k}(t)$ in the testing process of the single modality using triangular norms.

\section{Experiment Results}

\subsection{AMI Database}

In this paper, the Augmented Multi-Party Interaction (AMI) corpus were used for our experiment[15].The AMI corpus consists of audio-visual data captured of our participants in a natural meeting scenario. The participants volunteered their time freely and were assigned roles such as "project manager" or "marketing director" for the task of designing a new remote control device. The teams met over several sessions of varying lengths (15-35 minutes).

The meetings were not scripted and different activities were carried out such as presenting at a slide screen, explaining concepts on a whiteboard or discussing while sitting around a table. The participants therefore interacted naturally, including talking over each other. Data was collected in an instrumented meeting room. This contains a table, slide screen, white board and four chairs. While participants were requested to return to the same seat for the duration of a meeting session, they could move freely throughout the meeting. Different audio sources of varying distance to the speaker, and different video sources of varying views and fields-of-view represent audio-visual data of varying quality which is useful for robustness testing.

In this experiment, the subset of AMI database named AMIES2016 was used. For this experiment, we captured 5 video segments from each people's video that at last a total of 20 small video segments were obtained. We denoted it's as S1 to S20. For the reason of most of the image frames in the video have poor quality and no nose in the images, so we should delete it and then regular the image to guarantee nose is in the center of the image. Then select 10 frames from the video and record as 1 to 10 to construct the AMIES2016 face database. For each image, we normalized it to form the uniform size of $64 * 64$. Figure 1 show 10 frames selected from one video.

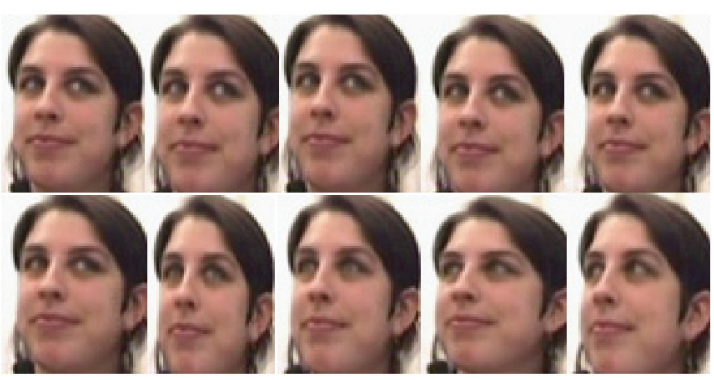

Fig.1. Face images of AMIES2016 database videos

\subsection{Experiment Overview}

The simulation experiments in this paper are conducted based on face recognition and speaker recognition. The face recognition experiments are conducted using the method proposed in [16-18], and the speaker recognition experiments are conducted using the 
method proposed in [19-20], and all are our preliminary works. We sign the face matcher as F1, F2 and F3, the speaker matcher as $\mathrm{S} 1$ and $\mathrm{S} 2$ respectively. The Equal Error Rate(EER) is used to assess the performance of the biometric systems, in biometric systems, we always try to make the EER smaller.

The experiments are include three parts:

(1) we compare the different triangular norm fusion methodologies using raw matching scores;

(2) we compare the fusion method proposed in this paper with different initial fusion strategies ;

(3) we compare the fusion method proposed in this paper with different fusion strategies which combined triangular norms.

\subsection{Compare the different triangular norm fusion methodologies}

In this part, we compare the performance between different triangular norm fusion methods using normalized matching scores. The triangular norm are proposed in section 2, and the performance of fusion can be adjudged by a GAR serving as one of the performance measures of biometric recognition. Its value equals $100-F R R$ and its represent the genuine accept rate .

In this experiments, the t-norm like Yager, Frank, Einstein and Hamacher are implemented for the score level fusion based on raw matching scores. The other fusion method by the SVM that require training and Sum, min, max rules which do not require any learning or training are also tested. The performance can be seen from Table 1.

Tab.1. The performance comparison between different score level fusion methods

\begin{tabular}{cc}
\hline Score level fusion methods for & GAR $(\%)$ \\
FAR $=0.01 \%$ & 93.8 \\
Yager t-norm & 94.6 \\
Frank t-norm & 94.25 \\
Einstein t-norm & 95 \\
Hamacher t-norm & 92.4 \\
SVM & 93.5 \\
Sum rule & 93 \\
Min rule & 92.43 \\
Max rule &
\end{tabular}

From the results shown in Table 1, it can be said that the fusion performance using t-norm are outperform than other classical score level fusion methods, the Yager $\mathrm{t}$-norm method is $93.8 \%$ and the Frank $\mathrm{t}$-norm is $94.6 \%$, especially the Hamacher t-norm fusion method, it is give the best performance with GAR $95 \%$ and it more than sum rule $1.5 \%$ and SVM method $2.6 \%$.

\subsection{Compare the new fusion method with different initial fusion strategies}

In this experiment, we measure the advantages of the proposed new fusion method over some initial score level fusion method such as LR, Tanh and MinMax with simple sum rule, these initial score level fusion methods are using the matching scores for the fusion step. The EER of all the single matcher can be found in Table 2 .

Tab. 2. EER of each simple recognition systems

\begin{tabular}{cccccc}
\hline & F1 & F2 & F3 & S1 & S2 \\
\hline EER(\%) & 6.1 & 4.1 & 3.5 & 2.8 & 4.45 \\
\hline
\end{tabular}

As shown in Table 2, among the three face matchers, the matcher F3 gain the best performance, and among the speech matchers, the performance order is S1, S2.

The fusion experiments are conducted with six multimodal combination. Table 3 shows the performance of multimodal fusion among the proposed new method named transfer function triangular fusion(TFTF), LR, Tanh and MaxMin (MM)with simple sum rule.

Tab.3. EER comparison between different fusion methods

\begin{tabular}{lcccccc}
\hline EER(\%) & F1S1 & F1S2 & F2S1 & F2S2 & F3S1 & F3S2 \\
\hline TFTF & 0.490 & 1.255 & 0.463 & 1.050 & 0.250 & 0.901 \\
LR & 0.628 & 1.560 & 0.500 & 1.000 & 0.293 & 0.985 \\
Tanh & 0.571 & 1.754 & 0.545 & 1.750 & 0.513 & 0.668 \\
MM & 0.571 & 1.638 & 0.916 & 1.264 & 0.476 & 1.250 \\
\hline
\end{tabular}

From the Table 3, we can observe that the proposed new method provides the best performance 4 times and the second performance 2 times, that means the proposed new fusion method gain the best performance. For example, the combination F3S1 give the best performance and the lowest EER is 0.250 , far lower than the single modality recognition.

In order to evaluate our method more precisely, we using rank mark to evaluate our fusion method. For example, the performance mark for the best matcher is 4 and followed is $3,2,1$. In the special circumstance, the 
performance of the two matchers are the same, that the mean value of the two mark are used for evaluate. Table 4 shows the performance mark of different fusion methods.

Tab.4. Performance mark comparison between different fusion methods

\begin{tabular}{|c|c|c|c|c|c|c|}
\hline Mark & F1S1 & F1S2 & F2S1 & F2S2 & F3S1 & $\mathrm{F} 3 \mathrm{~S} 2$ \\
\hline $\begin{array}{c}\text { TFT } \\
\text { F }\end{array}$ & 4 & 4 & 4 & 3 & 4 & 3 \\
\hline LR & 1 & 3 & 3 & 4 & 3 & 2 \\
\hline Tanh & 2.5 & 1 & 2 & 1 & 1 & 4 \\
\hline MM & 2.5 & 2 & 1 & 2 & 2 & 1 \\
\hline
\end{tabular}

From the results shown in Table 4, we can easily find that the TFTF method give the best recognition performance because the total mark is 22 and it is the largest one, the mark of the LR method is 16 and it is the second well performance.

\subsection{Compare the new fusion method with different initial fusion strategies which combine triangular norms}

In this experiment, we measure the advantages of the proposed new fusion method over some initial score level fusion method combined triangular norms, these fusion methods named MaxMin_T, Tanh_T, T_SVM.Table 5 shows the performance of multimodal fusion among these four fusion methods.

Tab.5. EER comparison between different fusion methods based on Triangular Norms

\begin{tabular}{ccccccc}
\hline EER(\%) & F1S1 & F1S2 & F2S1 & F2S2 & F3S1 & F3S2 \\
\hline TFTF & 0.490 & 1.255 & 0.463 & 1.050 & 0.250 & 0.901 \\
Tanh_T & 0.525 & 1.698 & 0.500 & 1.576 & 0.425 & 0.631 \\
MM_T & 0.525 & 1.360 & 0.423 & 0.692 & 0.454 & 0.988 \\
T_SVM & 1.327 & 2.250 & 0.800 & 1.131 & 0.500 & 1.020 \\
\hline
\end{tabular}

From the Table 5, we can see that the proposed fusion method still outperform than Tanh_T, MM_T and T_SVM methods, it is give the best performance 3 times and the second performance 3 times. And the EER of the Tanh_T and MM_T are lower than the Tanh and MM methods, so it prove that the triangular norms can improve the fusion performance certainly.

Table 6 shows the performance mark of different fusion methods based on triangular norms. From the Table 6, we can easily find that the total mark of TFTF is 21 , its the largest mark, the MM_T give the second performance and its total is 17.5 .
Tab. 6. Performance mark comparison between different fusion methods based on Triangular Norms

\begin{tabular}{ccccccc}
\hline Mark & F1S1 & F1S2 & F2S1 & F2S2 & F3S1 & F3S2 \\
\hline TFTF & 4 & 4 & 3 & 3 & 4 & 3 \\
Tanh_T & 2.5 & 2 & 2 & 1 & 3 & 4 \\
MM_T & 2.5 & 3 & 4 & 4 & 2 & 2 \\
T_SVM & 1 & 1 & 1 & 2 & 1 & 1 \\
\hline
\end{tabular}

\section{Conclusion}

The domain of multibiometric is a new and exciting area of information science and pattern recognition research. Recent years have seen a significant increase in research activity directed understanding all aspects of biometric information system representation and utilization for decision making support. This paper is specifically focused on understanding the complex mechanisms employed to find a good combination of multiple biometric traits and various fusion methods to get a optimal recognition results.

In this paper, a novel fusion scheme which using FAR, FRR and triangular norms is proposed. It firstly calculates FRR and FAR based on training samples, and obtained the Transfer function based on FAR and FRR. Then computes Transfer function of each matching scores through interpolation based on testing samples. At the last, the final decision are made by t-norms. Compared to the existing methods, the fusion algorithm proposed in this paper can greatly improve the recognition performance of multibiometric system compared to existing fusion methods.

Future work should focused on following two fields:

(1) The computation of the transfer function in the testing process is based on interpolation, the following work should investigate a new method to compute the optimal transfer function.

(2) The transfer function can fused by other complex fusion method and also its our future work. 


\section{Renferences}

1. Yong $\mathrm{Xu}$, Qi Zhu, David Zhang, Combine crossing matching scores with conventional matching scores for bimodal biometrics and face and palmprint recognition .Neurocomputing.74 (2011)3946-3952.

2. A. K. Jain, A. Ross, and S. Pankanti, "Biometrics: A tool for information security," IEEE Trans. Inf. Forensics Security, 1(2)..(2006) 125-143

3. Bahador Khaleghi, Alaa Khamis, Fakhreddine O., Saiedeh N. Razavi,Multisensor data fusion: A review of the state-of-the-art, Information Fusion ,14 (2013) 28-44.

4. B. Khaleghi, A. Khamis, F. Karray, Random finite set theoretic based soft/hard data fusion with application for target tracking, in: Proc. of the IEEE International Conference on Multisensor Fusion and Integration for Intelligent Systems, (2010) pp. 50-55.

5. M. P. Down and R. J. Sands, "Biometrics: An overview of the technology,challenges and control considerations," Inf. Syst. Control J. vol. 4, (2004) pp. 53-56.

6. A. Ross and R. Govindarajan, "Feature level fusion using hand and face biometrics," in Proc. SPIE 2nd Conf. Biometric Technol. Human Identification, Orlando, (2005) pp. 196-204.

7. Romain Giot,, Christophe Rosenberger, Genetic programming for multibiometrics, Expert Systems with Applications, 39 ,2012) 1837-1847.

8. Hanmandlu, M., Grover, J., Madasu, V.K., Vasirkala, S . Score level fusion of hand based biometrics using t-norms. In: IEEE International Conference on Technologies for Homeland Security (HST). Waltham, MA, (2010) pp. 70-76.

9. Klement, Erich Peter, Mesiar, Radko, Pap, Endre . Triangular Norms. Kluwer, Dordrecht, ISBN 0-7923-6416-3.(2000)
10. Kumar, A., Kanhangad, V., Zhang, D . A new framework for adaptive multimodal biometrics management. IEEE Trans. Inf. Forensics Secur. 5 (1),(2010) 92-102.

11. Ross A, Nandakumar K, Jain A K, Handbook of Multibiometrics, Springer, New York.

12. Adil Omari and Aníbal R. Figueiras,Feature Combiners With Gate Generated Weights for Classification.IEEE Transaction on Neural Networks and Learning Systems, 24(1) (2013) 158-163.

13. hang, L., Zhang, D., Zhu, H.Online finger-knuckle-print verification for personal authentication. Pattern Recognition. 43 (7) (2010) 2560-2571.

14. Jain A, Nandakumar K, Ross A. Score normalization in multimodal biometric systems. Pattern Recognition, 38(12) (2005) 2270-2285.

15. http://www.amiproject.org/.

16. Jie Cao,Di Wu.Face recognition based on discrimination power analysis and LDA-LPP algorithms.Journal of Jilin University (Engineering and Technology Edition),42(6) (2012) 1527-1531.

17. Di Wu,Jie Cao.Multi-Feature Fusion Face Recognition Based on KRWDA Algorithm Under Smart Environment.Journal of Jilin University (Engineering and Technology Edition),43(2) (2013) 439-443.

18. Di Wu,Jie Cao, Jinhua Wang,Wei Li,Multi-feature fusion face recognition based on Kernel Discriminate Local Preserve Projection Algorithm under smart environment.Journal of Computers.7(10) (2012) 2479-2487.

19. Di Wu,Jie Cao, Jinhua Wang.Speaker Recognition Based on Adapted Gaussian Mixture Model and Static and Dynamic Auditory Feature Fusion.Optics and Precision Engineering,21(6) (2013) 1615-1621.

20. Di Wu,Jie Cao, Jinhua Wang..Speaker Recognition Based on i-vector and Improved Local PreservingProjection.TELOCMNIKA,12(4) (2013) 335-360. 